\title{
Arterial Stiffness in Patients Taking Second-generation Antipsychotics
}

\author{
Ebru Fındıkıı ${ }^{1}$, Mustafa Gökçe ${ }^{2}$, Vedat Nacitarhan ${ }^{3}$, Mehmet Akif Camkurt ${ }^{4}$, Hüseyin Avni Fındıklı ${ }^{5}$, Selçuk \\ Kardaş $^{1}$, Merve Coşgun Şahin ${ }^{1}$, Mehmet Fatih Karaaslan ${ }^{1}$ \\ Departments of ${ }^{1}$ Psychiatry, ${ }^{2}$ Neurology, and ${ }^{3}$ Pyhsical Therapy and Rehabilitation, Faculty of Medicine, Kahramanmaraş Sütçü İmam \\ University, Kahramanmaraş, ${ }^{4}$ Department of Psychiatry, Afsin State Hospital, Kahramanmaraş, ${ }^{5}$ Department of Internal Medicine, Faculty \\ of Medicine, Adıyaman University, Adıyaman, Turkey
}

\begin{abstract}
Objective: That treatment with second-generation antipsychotics (SGAs) causes metabolic side effects and atherosclerosis in patients with schizophrenia and bipolar disorder (BD) is well-known. Increased arterial stiffness is an important marker of arteriosclerosis and has been identified as an independent risk factor for cardiovascular diseases. We measured pulse wave velocity (PWV) as a marker of arteriosclerosis in patients with schizophrenia and BD who use SGAs.

Methods: Patients and controls were collected from our psychiatry outpatient clinics or family medicine. Mental illness was diagnosed according to the Diagnostic and Statistical Manual of Mental Disorders, 4th edition. Mean age, gender, systolic and diastolic blood pressure, body mass index, Framingham risk score (FRS), etc. were determined. Simultaneous electrocardiography and pulse wave were recorded with an electromyography device. The photo-plethysmographic method was used to record the pulse wave. Inclusion criteria included use of SGAs for at least the last six months. Patients with diseases that are known to cause stiffness and the use of typical antipsychotics were excluded.

Results: Ninety-six subject (56 patients, 40 controls) were included in our study. There were 49 females, 47 males. Patients had schizophrenia $(n=17)$ and BD $(n=39)$. Their treatments were quetiapine $(n=15)$, risperidone $(n=13)$, olanzapine $(n=15)$, and aripiprazole $(n=13)$. Although differences in mean age, gender, and FRS in the patient and control groups were not statistically significant $(p=1)$, PW was greater in patients in the antipsychotic group ( $p=0.048)$.

Conclusion: This study supported the liability to stiffness in patients with schizophrenia and BD. Using SGAs may contribute to arterial stiffness in these patients.
\end{abstract}

KEY WORDS: Arterial stiffness; Pulse wave velocity; Schizophrenia; Bipolar disorder; Second-generation antipsychotics; Cardiovascular disorders.

\section{INTRODUCTION}

Compared with the general population, patients with serious mental illnesses such as schizophrenia (Sch) and bipolar disorder (BD) have a 25- to 30-year shorter life span that is not explained by suicide and accidents alone. ${ }^{1,2)}$ The main reason for increased risks of morbidity and mortality in these patients is cardiovascular diseases (CVDs) and stroke. ${ }^{3)}$ This excess of CVD- and stroke-related mortality may be due to a number of factors, including direct effects

\footnotetext{
Received: March 18, 2016 / Revised: April 12, 2016

Accepted: April 13, 2016

Address for correspondence: Ebru Findıkl1, MD Department of Psychiatry, Faculty of Medicine, Kahramanmaraş Sütçü İmam University, 46100, Kahramanmaraş, Turkey Tel: +90-3442803367, Fax: +90-3442803409

E-mail: ebrukanmaz@gmail.com

This study is presented as a poster presentation in 7th Internationa Congress on Psychopharmacology and pressed in Bulletin of Clinical Psychopharmacology 2015;25(suppl 1):P63-64.
}

of their illness, medications used to treat the illness, modifiable behavioral risk factors (e.g., smoking, obesity, and physical inactivity). ${ }^{4)}$ Adverse effects of psychotropic drugs also may contribute to the development of CVDs and stroke by increasing the risk of conditions such as hyperglycemia, hyperlipidemia, and obesity. ${ }^{57)}$

Second-generation antipsychotics (SGAs) have been widely used as supposedly safer and better-tolerated alternatives to first-generation antipsychotics for the treatment of Sch, BD, and behavioral and psychological symptoms of dementia and delirium since the 1990s. ${ }^{8)}$ Over time, with their increased use, an increased risk of CVD and stroke from olanzapine and risperidone were reported. ${ }^{9-12)}$ A recent systematic review showed conflicting findings regarding the cerebrovascular safety of risperidone. ${ }^{13)}$ Additionally, data from a clinical trial using quetiapine to treat behavioral and psychological symptoms of dementia showed no increased risk of CVD compared with a placebo. ${ }^{14,15)}$

() This is an Open-Access article distributed under the terms of the Creative Commons Attribution Non-Commercial License (http://creativecommons.org/licenses/by-nc/4.0) which permits unrestricted non-commercial use, distribution, and reproduction in any medium, provided the original work is properly cited. 
Increased arterial stiffness is an important marker of arteriosclerosis and has been identified as an independent risk factor for CVD. ${ }^{16)}$ Over time, the artery wall constantly loses elasticity and becomes rigid. ${ }^{17-19)}$ Hypertension and other diseases like diabetes mellitus (DM) accelerate this process. ${ }^{20)}$ Several indices have been developed to characterize arterial stiffness; pulse wave velocity (PWV) is the most recognized and established index of arterial stiffness because it is very evidential and measurable by commercially available devices. ${ }^{15,19,20)}$ The parameter of real spreading of pulse waves in the artery system is PWV or pulse transit time (PTT), which is related according to the formula $\mathrm{PWV}=\mathrm{L} / \mathrm{PTT}$. $\mathrm{L}$ is the distance between two points between which the velocity is measured. ${ }^{21)}$ An increased PWV and a relatively short PTT are observed with high blood pressure, aging, arteriosclerosis, and DM. ${ }^{18,22)}$

To date, uncertainty in the causes and mechanisms is continuing over increased CVD and stroke in psychiatric patients using SGAs. One of the possible mechanisms is increased arterial stiffness. In this study, we aimed to measure arterial stiffness using PWV in patients with Sch and BD who were given SGAs.

\section{METHODS}

\section{Participants}

Ninety-six subject (56 patients, 40 controls) were included in our study. Fifty were female and 47 were men. Subjects were collected from our outpatient clinics of psychiatry (psychiatric patients) or family medicine (control subjects). Mental illness was diagnosed according to the Diagnostic and Statistical Manual of Mental Disorders, 4th edition (DSM-IV). Patients had Sch $(n=17)$ and BD $(n=39)$. Their treatments were quetiapine $(n=15)$, risperidone $(n=13)$, olanzapine $(n=15)$, and aripiprazol $(n=13)$ for at least the last six months. Age- and sex-matched controls had no psychiatric or physical illness history and were not on any medicine treatment. Subjects who had risk factors for arterial stiffness, such as unstable angina, myocardial infarcts, hypertension, stroke, DM, etc., and were relatively older (age $>55$ years old) were excluded. In addition, patients who had collagen vascular disease, used together typical and atypical antipsychotics, had a duration of antipsychotic treatment less than six months, and alcohol abuse, substance abuse, or smoking were excluded. This study was approved by our institutional ethical committee, and informed consent was obtained prior to participation in the study (2013/14-10).

\section{Subject Assessments and Clinical and Laboratory Measurements}

Socio-demographic and clinical information was collected either from participants and their relatives or the electronic medical record of Department of Psychiatry, Faculty of Medicine, Kahramanmaraş Sütçü İmam University (Kahramanmaraş, Turkey). Clinical characteristics, such as age of onset and duration of illness, were ascertained by direct questioning. Current use of medications at the time of interview was recorded, and detailed medication histories were obtained through systematic review of all pharmacy records for the previous five years.

Fasting plasma lipids (total cholesterol, triglycerides, low-density lipoprotein cholesterol, and high-density lipoprotein cholesterol [HDL-C]) were determined at the department of biochemistry of our hospital. Height, weight, body mass index (BMI), and systolic and diastolic blood pressure were assessed at the time of electrophysiological measurement. Blood pressures were measured in the seated position using an automated sphygmomanometer (HEM-907; Omron, Osaka, Japan).

The Framingham risk score (FRS) (D'Agostino et $a l^{23)}$ ) provides a percentage estimate of predicted 10-year CVD risk. FRS was calculated according to the formula developed by Wilson et al. ${ }^{24)}$ (see http://cvdrisk.nhlbi.nih. gov/calculator.asp). It is calculated using clinical variables including sex, age, total cholesterol, HDL-C, smoker or not, and on antihypertensive or not. FRS was grouped in the following categories: Group 1, FRS $<10 \%$; Group 2, FRS $\geq 10 \%$ and $<20 \%$; and Group 3 , FRS $\geq 20 \%$.

\section{Electrophysiological Assessment}

Before the experiments, subjects abstained from alcohol and caffeine and fasted for $>3$ hours. Subjects were studied under supine resting conditions in a quiet, temperature-controlled $\left(22^{\circ} \mathrm{C}\right.$ to $\left.24^{\circ} \mathrm{C}\right)$ room. Measurements were performed after at least 10 minutes supine rest.

The electrocardiogram (ECG) and pulse wave were recorded using the Neuro-MEP-Micro (ver. 2009) electromyography (EMG) device (Neurosoft Medical Diagnostic Equipment, Ivanovo, Russia). The sampling rate of the Neuro-MEP-Micro EMG device was adjusted to 1,000 Hz. The lower cut-off frequency and the uppercut-off frequency were chosen as $0.01 \mathrm{~Hz}$ and $100 \mathrm{~Hz}$, respectively. A 50-Hz band suppression filter was used. The first channel was used for ECG recordings, and the second channel was used to record pulse wave (Fig. 1A).

ECG recordings were performed by using standard bipolar limb lead-I (negative electrode on the back of the 


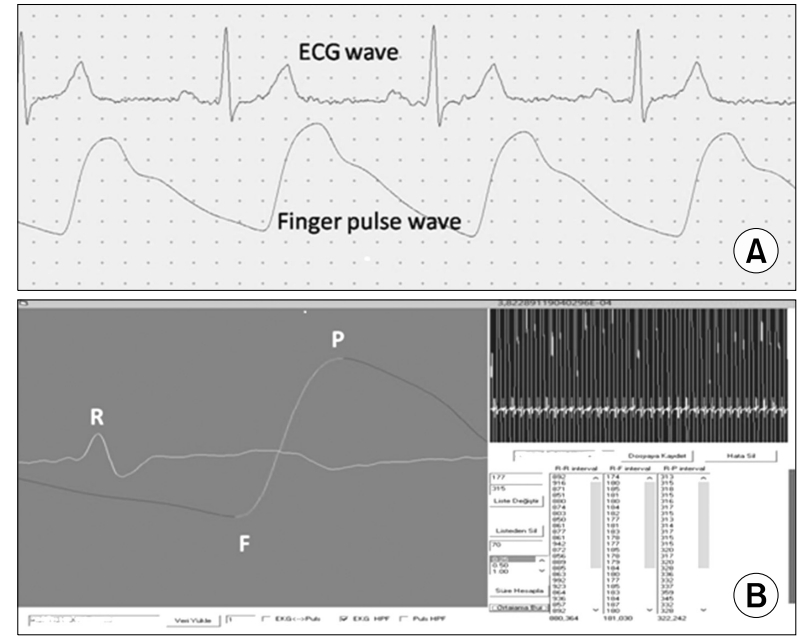

Fig. 1. (A) The first line is electrocardiogram recordings (ECG wave); and the second line is the pulse wave. They were synchronized on the screen of the EMG device. (B) The ECG and pulse wave was superimposed by a computer program. The program automatically calculated an average value from 30 different measurements. The 30 different values from each subject were seen on the screen (you can see the right-bottom part on this figure), and we visually checked all measurements to prevent any mistakes.

$R$, R wave of ECG; $F$, foot; $P$, peak.

right hand, positive electrode on the back of the left hand, the ground electrode on the left foot) with the first channel of the EMG device. The photo-plethysmographic method was used to record the pulse wave. The pulse wave was recorded with adult-type $\mathrm{SpO} 2$ pulse oximeter probe (Nihon-Kohden brands, Tokyo, Japan) with an external power supply. A high-pass resistance-capacitor filter (1 MOhm and $0.22 \mu \mathrm{F}$ for $0.7 \mathrm{~Hz}$ ) was used on the probe's sensor output. The filter output was attenuated by resistance and was connected to the second channel of the EMG device. The pulse wave that was detected from the fingertip by the pulse oximeter probe attached to the right-hand index finger was recorded by the EMG device.

In order to evaluate data, the synchronized recorded ECG and pulse wave traces were exported as a file using the export feature of the EMG device. The R-wave peak points on the ECG trace and onset of the ascending limb ( $\mathrm{F}$, foot point) and peak point $(\mathrm{P})$ on the pulse wave were determined with a custom detection algorithm. The R-wave peak points in the ECG traces were considered as the beginning of the pulse wave. The period between the peak of the R wave in the ECG (first channel) and the starting point of the ascending limb and peak of pulse waves recorded from the fingertip (second channel) were measured automatically (Fig. 1). The detected R waves, foot points, and peak points were revised visually against possible measurement error. The time (ms) between R and foot point (RF-PTT) was considered the PTT, and the foot and peak time was considered as a rise time (RP-PTT). The distance between the sternal notch and the index finger pulp was measured in millimeters while the shoulder was is in the abduction position. The upper-limb PWV $(\mathrm{m} / \mathrm{s})$ was calculated by dividing the distance $(\mathrm{mm})$ by PTT (ms).

\section{Statistical Analysis}

For the analysis, the SPSS software package for Windows (version 16.0; SPSS Inc., Chicago, IL, USA) was used. Study data were summarized by using descriptive statistics (e.g., frequency, percentage). Patient and control groups were compared by independent Student $t$-test. One-way ANOVA was used for comparing Sch, BD, and control. In addition, the ANOVA test was used to compare the sub-treatment groups. The statistical level of significance was defined as $p<0.05$.

\section{RESULTS}

A total of 96 subjects (56 patients and 40 controls) were included in the study. The demographic and fundamental data of participants are shown in Table 1. The subjects in the psychiatric disorders group consisted of Sch $(n=17)$ and $\operatorname{BD}(n=39)$. The average durations of illness and medication were 5.8 and 2.18 years, respectively. The range of doses of risperidone, olanzapine, quetiapine, and aripiprazole were 2-6 mg, 5-20 mg, 200-800 mg, and 10-30 mg, respectively. Sixteen of BD patients were on mood stabilizer medication ( $41.0 \%$ of BD patients).

The mean age was 37.14 years for patients versus 36.38 years for controls $(p=0.688)$. Gender differences and BMI in the patient and control groups were also statistically insignificant ( $p=1$ and 0.125 , respectively). According to FRS, patients were 50/56 in Group 1, 4/56 in Group 2, and $1 / 56$ in Group 3. The FRS for all control subjects were in Group 1. The differences between the patient and control groups were not statistically significant (Table 1).

PWV was higher in the patient group than in the control group ( $p=0.048$ ). RF-PTT (patients, $171 \mathrm{~ms}$; controls, 176 $\mathrm{ms}$ ) and RP-PTT (patients, $337 \mathrm{~ms}$; controls, $348 \mathrm{~ms}$ ) were shorter in the patient group than in the control group. However, these differences did not reach statistical significance (Table 1).

When we did patient subgroup analysis, no statistically significant difference was found according to PWV, 
Table 1. Vascular indices of patients and healthy control groups

\begin{tabular}{|c|c|c|c|}
\hline Variable & Patient $(n=56)$ & Control $(n=40)$ & $p$ value \\
\hline Age $(y r)$, mean & 37.14 & 36.38 & $0.688^{*}$ \\
\hline \multicolumn{4}{|l|}{ Sex } \\
\hline Male & $29(51.8 \%)$ & $18(45.0 \%)$ & $1^{\dagger}$ \\
\hline Female & 27 (48.2\%) & $22(55.0 \%)$ & \\
\hline Height $(\mathrm{cm})$ & 168.1 & 167.9 & $0.924^{*}$ \\
\hline Weight (kg) & 82.2 & 77.5 & $0.112^{*}$ \\
\hline BMI $\left(\mathrm{kg} / \mathrm{m}^{2}\right)$ & 29.1 & 27.5 & $0.125^{\star}$ \\
\hline $\mathrm{HDL}(\mathrm{mg} / \mathrm{dl})$ & 46.8 & 44.1 & $0.337^{*}$ \\
\hline Total cholesterol (mg/dl) & 197 & 182 & $0.101^{*}$ \\
\hline FRS & & & $1^{\dagger}$ \\
\hline Group 1 & 54 & 40 & \\
\hline Group 2 & 2 & - & \\
\hline Group 3 & 1 & - & \\
\hline SBP (mmHg) & 123 & 118.5 & $0.057^{*}$ \\
\hline $\mathrm{DBP}(\mathrm{mmHg})$ & 81 & 74 & $0.001^{*}$ \\
\hline RF-PT (ms) & 171.5 & 176.6 & $0.072^{*}$ \\
\hline RP-PTT (ms) & 337.2 & 348.6 & $0.217^{*}$ \\
\hline PWV (cm/s) & 4.91 & 4.73 & $0.048^{*}$ \\
\hline
\end{tabular}

BMI, body mass index; HDL, high density lipoprotein; FRS, Framingham risk score; SBP, systolic blood pressure; DBP, diastolic blood pressure; RF-PTT, R-foot pulse transit time; RP-PT,, R-peak pulse transit time; PWV, pulse wave velocity.

$p$ values by ${ }^{*}$ Student $t$-test and ${ }^{\dagger}$ chi-square test.

Table 2. Pulse wave parameters of treatment groups

\begin{tabular}{cccccc}
\hline Parameter of pulse wave & Risperidone $(n=13)$ & Olanzapine $(n=15)$ & Quetiapine $(n=15)$ & Aripiprazole $(n=13)$ & $p$ value \\
\hline RF-PTT (ms) & 167 & 167 & 170 & 172 & 0.218 \\
RP-PTT (ms) & 332 & 329 & 339 & 348 & 0.546 \\
PWV (cm/sec) & 4.83 & 4.94 & 5.00 & 4.80 & 0.206 \\
\hline
\end{tabular}

RF-PTT, R-foot pulse transit time; RP-PTT, R-peak pulse transit time; PWV, pulse wave velocity.

$p$ value by ANOVA.

RF-PTT, and RP-PTT in Sch and BD. In addition, in our analysis according to treatment group (risperidone, olanzapine, quetiapine, and aripiprazole), there were no statistically significant differences between these four treatment groups (Table 2).

\section{DISCUSSION}

This is the first study investigating the impact of SGA therapy on arterial stiffnes measured as upper-limb PWV in patients with Sch and BD. PWV was found to be significantly faster in patients who used antipsychotics than those in the control group. This finding actually indicates arterial stiffness in these patients and correlated with the main body of published literature in the general meaning. ${ }^{25-27)}$ Arterial hypertension as a main arterial stiffness indicator was higher in the patient groups than in the control group. Although both RF-PTT and RP-PTT were shorter in the patient group, they did not reach statistical significance.

Sch and BD are known as independent risk factors for metabolic syndrome. ${ }^{25,27)}$ A complex combination of behavioral (smoking, alcohol misuse, etc.) and pathophysiological mechanisms (autonomic dysfunction, decreased heart rate variability, endothelial dysfunction increased platelet reactivity, and abnormal immune system activation) are the main causes of CVD and stroke in patients with psychiatric illness. ${ }^{28)}$ Given the predictive power of PWV, identifying strategies that prevent or reduce stiffening may be important in the prevention of cardiovascular events in psychiatric patients. ${ }^{29)}$ However, there are limited studies about arterial stiffness and PWV in severe psychiatric illnesses that supported increased $\mathrm{PWV}$ in patients with Sch and BD. ${ }^{30,31)}$ In our study, the reason for increased PWV in the patient group may be related to their primary psychiatric disease and/or SGAs and further illness or conditions.

To our knowledge, there is no investigation comparing the effect of SGAs to arterial stiffness and PWV measurement in patients with Sch and BD. Only one investigation -Fiedorowicz et al. $^{32)}$ - suggested that first-generation 
antipsychotic exposure was associated with arterial stiffness, although SGAs were associated with dyslipidemia and insulin resistance but not arterial stiffness. No detailed information about subgroup medication effect on arterial stiffness was given in their study. Aripiprazole and ziprasidone are defined as low-metabolic risk agents, olanzapine was classified as having higher metabolic risk, and quetiapine and risperidone were classified as having discrepant metabolic risk for diabetes and worsening lipid profiles. $^{25,33)}$ It has been suggested that antipsychotic drugs produce direct effects on the heart and blood vessels via the blockade of adrenergic and cholinergic receptors, as well as indirect effects through central autonomic regulation and baroreceptor reflexes. ${ }^{34,35)}$ These autonomic actions are thought to mediate most of the cardiovascular side effects of antipsychotic drugs, such as tachycardia and increased blood pressure. In our study, when pulse wave parameters (PWV, RF-PTT, RP-PTT) were analyzed according to medication subgroups, in the aripiprazole group, PWV and PTT values were similar to controls, but all four medication subgroups showed no statistically significant difference between groups. The small sample size of these subgroups may affect the significance of our results.

In our study, the FRS of most patients (50/57 patients) were in Group 1, and a few patients were in other groups. According to FRS, there were no statistically significant differences in the patient and control groups. Although an increased PWV was found in the patient group, cardiovascular risk was less than $10 \%$ in our patients according to FRS. It is known that arterial stiffness is an independent predictor of cardiovascular risk, from current established risk factors, or even the FRS. ${ }^{36)}$ However, our study is cross-sectional, and it can be said that arterial stiffness measurement is an independent predictor of CVD in psychiatric patients.

There are some natural limitations of our study. A natural limitation is that there was no drug-naive group. Drug-naive patient groups might give us the ability to explain the drug effect on arterial stiffness independent from illness effect. The other limitation is that the diseases (Sch and BD) themselves might affect arterial stiffness, as could many other factors (acutely or chronically).

In conclusion, SGAs are used worldwide for the treatment of various psychiatric disorders. The association between arterial stiffness and the use of SGAs is not well-established yet. In this study, we showed that using atypical antipsychotic medications may cause arterial stiffness in Sch and $\mathrm{BD}$, but confounding factors may change our results.

\section{Acknowledgments}

We thank Dr. Adem Doganer from the Department of Biostatistics, Faculty of Medicine, Kahramanmaraş Sütçü İmam University, for his gentle contribution to our manuscript.

\section{REFERENCES}

1. Harris EC, Barraclough B. Excess mortality of mental disorder. Br J Psychiatry 1998;173:11-53.

2. Brown AS, Birthwhistle J. Excess mortality of mental illness. Br J Psychiatry 1996;169:383-384.

3. Newcomer JW. Medical risk in patients with bipolar disorder and schizophrenia. J Clin Psychiatry 2006;67:el6.

4. Gierisch JM, Nieuwsma JA, Bradford DW, Wilder CM, Mann-Wrobel $\mathrm{MC}$, McBroom $\mathrm{AJ}$, et al. Interventions to improve cardiovascular risk factors in people with serious mental illness. AHRQ Comparative Effectiveness Reviews. Rockville, MD:Agency for Healthcare Research and Quality (US);2013.

5. Newcomer JW. Metabolic considerations in the use of antipsychotic medications: a review of recent evidence. $J$ Clin Psychiatry 2007;68 Suppl 1:20-27.

6. Udomratn P. Metabolic syndrome in psychiatric patients treated with antipsychotic drugs in Thailand. Clin Psychopharmacol Neurosci 2010;8:79-83.

7. Ko YK, Soh MA, Kang SH, Lee JI. The prevalence of metabolic syndrome in schizophrenic patients using antipsychotics. Clin Psychopharmacol Neurosci 2013;11:80-88.

8. Horacek J, Bubenikova-Valesova V, Kopecek M, Palenicek $\mathrm{T}$, Dockery C, Mohr P, et al. Mechanism of action of atypical antipsychotic drugs and the neurobiology of schizophrenia. CNS Drugs 2006;20:389-409.

9. McConville BJ, Sorter MT. Treatment challenges and safety considerations for antipsychotic use in children and adolescents with psychoses. J Clin Psychiatry 2004;65 Suppl 6:20-29.

10. Mowat D, Fowlie D, MacEwan T. CSM warning on atypical psychotics and stroke may be detrimental for dementia. BMJ 2004;328:1262.

11. Wooltorton E. Olanzapine (Zyprexa): increased incidence of cerebrovascular events in dementia trials. CMAJ 2004;170: 1395.

12. Shin JY, Choi NK, Lee J, Seong JM, Park MJ, Lee SH, et al. Risk of ischemic stroke associated with the use of antipsychotic drugs in elderly patients: a retrospective cohort study in Korea. PLoS One 2015;10:e0119931.

13. Mazzucco S, Cipriani A, Barbui C, Monaco S. Antipsychotic drugs and cerebrovascular events in elderly patients with dementia: a systematic review. Mini Rev Med Chem 2008;8:776-783.

14. Tariot PN, Schneider L, Katz IR, Mintzer JE, Street J, Copenhaver M, et al. Quetiapine treatment of psychosis associated with dementia: a double-blind, randomized, placebo-controlled clinical trial. Am J Geriatr Psychiatry 2006; 14:767-776.

15. Zhong KX, Tariot PN, Mintzer J, Minkwitz MC, Devine NA. Quetiapine to treat agitation in dementia: a randomized, double-blind, placebo-controlled study. Curr Alzheimer Res 2007;4:81-93. 
16. Hodes RJ, Lakatta EG, McNeil CT. Another modifiable risk factor for cardiovascular disease? Some evidence points to arterial stiffness. J Am Geriatr Soc 1995;43:581-582.

17. Kelly R, Hayward C, Avolio A, O'Rourke M. Noninvasive determination of age-related changes in the human arterial pulse. Circulation 1989;80:1652-1659.

18. Nichols WW. Clinical measurement of arterial stiffness obtained from noninvasive pressure waveforms. Am J Hypertens 2005;18(1 Pt 2):3S-10S.

19. Vaitkevicius PV, Fleg JL, Engel JH, O'Connor FC, Wright $\mathrm{JG}$, Lakatta LE, et al. Effects of age and aerobic capacity on arterial stiffness in healthy adults. Circulation 1993;88: 1456-1462.

20. Brooks B, Molyneaux L, Yue DK. Augmentation of central arterial pressure in type 1 diabetes. Diabetes Care 1999;22:1722-1727.

21. Korpas D, Hálek J, Dolezal L. Parameters describing the pulse wave. Physiol Res 2009;58:473-479.

22. O'Rourke MF. Isolated systolic hypertension, pulse pressure, and arterial stiffness as risk factors for cardiovascular disease. Curr Hypertens Rep 1999;1:204-211.

23. D'Agostino RB Sr, Vasan RS, Pencina MJ, Wolf PA, Cobain $\mathrm{M}$, Massaro JM, et al. General cardiovascular risk profile for use in primary care: the Framingham Heart Study. Circulation 2008;117:743-753.

24. Wilson PW, D'Agostino RB, Levy D, Belanger AM, Silbershatz H, Kannel WB. Prediction of coronary heart disease using risk factor categories. Circulation 1998;97: 1837-1847.

25. Haupt DW. Differential metabolic effects of antipsychotic treatments. Eur Neuropsychopharmacol 2006;16 Suppl 3:S149-S155.

26. Newcomer JW, Hennekens CH. Severe mental illness and risk of cardiovascular disease. JAMA 2007;298:1794-1796.

27. Taylor V, MacQueen G. Associations between bipolar disorder and metabolic syndrome: A review. J Clin Psychiatry 2006; 67:1034-1041.

28. Sowden GL, Huffman JC. The impact of mental illness on cardiac outcomes: a review for the cardiologist. Int $J$ Cardiol 2009;132:30-37.

29. Cecelja M, Chowienczyk P. Role of arterial stiffness in cardiovascular disease. JRSM Cardiovasc Dis 2012;1:cvd. 2012.012016.

30. Phillips AA, Warburton DE, Flynn SW, Fredrikson D, Lang DJ. Assessment of arterial stiffness among schizophreniaspectrum disorders using aortic pulse wave velocity and arterial compliance: a pilot study. Psychiatry Res 2014;215: 14-19.

31. Sodhi SK, Linder J, Chenard CA, Miller del D, Haynes WG, Fiedorowicz JG. Evidence for accelerated vascular aging in bipolar disorder. J Psychosom Res 2012;73:175-179.

32. Fiedorowicz JG, Coryell WH, Rice JP, Warren LL, Haynes WG. Vasculopathy related to manic/hypomanic symptom burden and first-generation antipsychotics in a sub-sample from the collaborative depression study. Psychother Psychosom 2012;81:235-243.

33. Newcomer JW. Second-generation (atypical) antipsychotics and metabolic effects: a comprehensive literature review. CNS Drugs 2005;19 Suppl 1:1-93.

34. Sugiyama A, Satoh Y, Hashimoto K. In vivo canine model comparison of cardiohemodynamic and electrophysiological effects of a new antipsychotic drug aripiprazole (OPC-14597) to haloperidol. Toxicol Appl Pharmacol 2001;173:120-128.

35. Mackin P. Cardiac side effects of psychiatric drugs. Hum Psychopharmacol 2008;23 Suppl 1:3-14.

36. Boutouyrie P, Tropeano AI, Asmar R, Gautier I, Benetos A, Lacolley $\mathrm{P}$, et al. Aortic stiffness is an independent predictor of primary coronary events in hypertensive patients: a longitudinal study. Hypertension 2002;39:10-15. 